MATHEMATICS OF COMPUTATION

Volume 80, Number 274, April 2011, Pages 1123-1133

S $0025-5718(2010) 02415-9$

Article electronically published on September 9, 2010

\title{
EQUIVARIANT GRÖBNER BASES AND THE GAUSSIAN TWO-FACTOR MODEL
}

\author{
ANDRIES E. BROUWER AND JAN DRAISMA
}

\begin{abstract}
Exploiting symmetry in Gröbner basis computations is difficult when the symmetry takes the form of a group acting by automorphisms on monomials in finitely many variables. This is largely due to the fact that the group elements, being invertible, cannot preserve a term order. By contrast, inspired by work of Aschenbrenner and Hillar, we introduce the concept of equivariant Gröbner basis in a setting where a monoid acts by homomorphisms on monomials in potentially infinitely many variables. We require that the action be compatible with a term order, and under some further assumptions derive a Buchberger-type algorithm for computing equivariant Gröbner bases.

Using this algorithm and the monoid of strictly increasing functions $\mathbb{N} \rightarrow \mathbb{N}$ we prove that the kernel of the ring homomorphism

$$
\mathbb{R}\left[y_{i j} \mid i, j \in \mathbb{N}, i>j\right] \rightarrow \mathbb{R}\left[s_{i}, t_{i} \mid i \in \mathbb{N}\right], y_{i j} \mapsto s_{i} s_{j}+t_{i} t_{j}
$$

is generated by two types of polynomials: off-diagonal $3 \times 3$-minors and pentads. This confirms a conjecture by Drton, Sturmfels, and Sullivant on the Gaussian two-factor model from algebraic statistics.
\end{abstract}

\section{InTRODUCTION AND RESUlts}

Equivariant Gröbner bases. Algebraic varieties arising from applications often have many symmetries. When analysing such varieties with tools from computational algebra, it is desirable to do so in an equivariant manner, that is, while keeping track of those symmetries, and if possible exploiting them. The notion of Gröbner basis, which lies at the heart of computational algebra, depends heavily on choices of coordinates and of a term order, which is a well-order on monomials in the coordinates. It is therefore natural, at least from a computational point of view, to study symmetries of ideals that preserve both the coordinates and the term order. Now the term symmetry is usually reserved for certain invertible maps, but it is easy to see that an invertible map cannot preserve a well-order; see Remark 2.1 . Hence we are led to relax the condition that symmetries be invertible. On the other hand, if a non-invertible map is to preserve the restriction of the term order to the set of coordinates, then that set better be infinite, in contrast with the usual set-up in computational commutative algebra.

Received by the editor August 11, 2009 and, in revised form, February 3, 2010.

2010 Mathematics Subject Classification. Primary 13P10, 16W22; Secondary 62H25.

Key words and phrases. Equivariant Gröbner bases, algebraic factor analysis.

The second author is supported by DIAMANT, an NWO mathematics cluster. 
In fact, there is another, more compelling reason for allowing infinitely many variables: many varieties from applications come in infinite families, and it is convenient to pass to a suitable limit. For example, the variety of symmetric $n \times n$ matrices of rank 2 has a well-defined projective limit for $n$ tending to infinity, and so does the closely related two-factor model that we study in this paper. In both cases, the limit is not only stable under the union of all symmetric groups, $S_{n}$, simultaneously permuting rows and columns, but also under the monoid $\operatorname{Inc}(\mathbb{N})$ of all strictly increasing maps from $\mathbb{N}$ to itself. And while the union of the symmetric groups does not preserve any term order, the monoid $\operatorname{Inc}(\mathbb{N})$ does preserve such an order; this fundamental observation allows us to do computations in Section 3 .

This discussion leads to the following set-up, which we believe will have applications to numerous other problems. Let $X$ be a potentially infinite set, whose elements we call variables. The free commutative monoid generated by $X$ is denoted Mon; its elements are called monomials. Suppose that we have

EGB1. a term order, i.e., a well-order $\leq$ on Mon such that $m \leq m^{\prime} \Rightarrow m m^{\prime \prime} \leq$ $m^{\prime} m^{\prime \prime}$ for all $m, m^{\prime}, m^{\prime \prime} \in$ Mon; and

EGB2. a monoid $G$, i.e., a (typically non-commutative) semigroup with identity, acting on Mon by means of monoid homomorphisms Mon $\rightarrow$ Mon preserving the strict order: $\pi(1)=1, \pi\left(m m^{\prime}\right)=\pi(m) \pi\left(m^{\prime}\right)$, and $m<m^{\prime} \Rightarrow$ $\pi m<\pi m^{\prime}$ for all $\pi \in G, m, m^{\prime} \in$ Mon.

Example 1.1. The setting that Aschenbrenner and Hillar study in [1 fits into this framework, and indeed inspired our set-up. There $X=\left\{x_{1}, x_{2}, \ldots\right\}$ and $G$ is the monoid Inc $(\mathbb{N})$ of all strictly increasing maps $\pi: \mathbb{N} \rightarrow \mathbb{N}$ acting on $X$ by $\pi x_{i}=x_{\pi(i)}$ and on Mon by multiplicativity. As a term order one can choose the lexicographic order with $x_{i}>x_{j}$ if $i>j$. Aschenbrenner and Hillar have turned their proof of finite generation of $\operatorname{Inc}(\mathbb{N})$-stable ideals in $K\left[x_{1}, x_{2}, \ldots\right]$ into an algorithm; see [2].

Let $K$ be a field and let $K[X]=K$ Mon be the polynomial $K$-algebra in the variables $X$, or, equivalently, the monoid $K$-algebra of Mon. Then $G$ acts naturally on $K[X]$ by means of homomorphisms. A $G$-orbit is a set of the form $G z=\{\pi z \mid$ $\pi \in G\}$, where $z$ is in a set on which $G$ acts. The fact that $G$ acts by monoid homomorphisms on Mon implies that the ideal generated by a union of $G$-orbits in $K[X]$ is automatically $G$-stable, that is, closed under the action of $G$.

We use the notation $\operatorname{lm}(f)$ for the leading monomial of $f$, i.e., the $\leq$-largest monomial having non-zero coefficient in $f$. By the requirement that $G$ preserve the order, we have $\operatorname{lm}(\pi f)=\pi \operatorname{lm}(f)$. Given an ideal $I$ of $K[X], \operatorname{lm}(I)$ is an ideal in the monoid Mon, that is, $\operatorname{lm}(I)$ is closed under multiplication with any element from Mon. If $I$ is $G$-stable, then so is $\operatorname{lm}(I)$.

Definition 1.2 (Equivariant Gröbner basis). Let $I$ be a $G$-stable ideal in $K[X]$. A $G$-Gröbner basis of $I$ is a subset $B$ of $I$ for which $\operatorname{lm}(G B)(=\{\operatorname{lm}(\pi b) \mid b \in B, \pi \in$ $G\}$ ) generates the ideal $\operatorname{lm}(I)$ in Mon. If $G$ is fixed in the context, we also call $B$ an equivariant Gröbner basis. If $G=\{1\}$, then we call $B$ an ordinary Gröbner basis.

Remark 1.3. At MEGA 2009, Viktor Levandovskyy pointed out to the second author that our equivariant Gröbner bases are a special case of Gröbner $S$-bases in the sense of [8], which were invented for analysing certain two-sided ideals in free associative algebras. The focus of the present article is on getting exactly the right set-up for doing machine computations of equivariant Gröbner bases in the commutative setting. 
It is easy to see that if $B$ is a $G$-Gröbner basis of $I$, then $G B$ generates $I$ as an ideal; see Lemma 2.2 .

Example 1.4. Let $X=\left\{y_{i j} \mid i, j \in \mathbb{N}\right\}$, let $k$ be a natural number, and let $I$ be the ideal of all polynomials in the $y_{i j}$ that vanish on all $\mathbb{N} \times \mathbb{N}$-matrices $y$ of rank at most $k$. Order the variables $y_{i j}$ lexicographically by the pair $(i, j)$, where $i$ is the most significant index; so for instance $y_{3,5}>y_{2,6}>y_{2,4}>y_{1,10}$. The corresponding lexicographic order on monomials in the $y_{i j}$ is a well-order. Let $G:=\operatorname{Inc}(\mathbb{N}) \times \operatorname{Inc}(\mathbb{N})$ act on $X$ by $(\pi, \sigma) y_{i j}=y_{\pi(i), \sigma(j)}$; this action preserves the strict order. The $G$-orbit of the determinant $D$ of the matrix $\left(y_{i j}\right)_{i, j=1, \ldots, k+1}$ consists of all $(k+1) \times(k+1)$ minors of $y$, which by the results of [12] form a Gröbner basis of the ideal $I$. As a consequence, $\{D\}$ is a $G$-Gröbner basis of $I$.

A $G$-stable ideal need not have a finite $G$-Gröbner basis. Indeed, if one requires that every $G$-stable ideal $I$ in $K[X]$ has a finite $G$-Gröbner basis, then this must, in particular, be true for monomial ideals. This implies that Mon does not have infinite antichains relative to the partial order on Mon defined by $m \preceq m^{\prime}: \Leftrightarrow \exists \pi \in G$ : $\pi m \mid m^{\prime}$. Observe that this is, indeed, a partial order: transitivity is straightforward, and antisymmetry follows from the fact that $\pi(m) \mid m \Rightarrow \pi(m) \leq m$; while on the other hand, $\pi(m) \geq m$ for all $\pi, m$; see Remark 2.1. Conversely, if (Mon, $\preceq$ ) does not have infinite anti-chains, then every ideal has a finite $G$-Gröbner basis. This is the case in the set-up of Example 1.1, which is generalised in [11; there equivariant Gröbner bases are called monoidal Gröbner bases.

Remark 1.5. We have not yet really used that Mon is the free commutative monoid generated by $X$. So far, we could have taken Mon as any commutative monoid equipped with EGB1 and EGB2. This viewpoint, and a generalisation thereof, is adopted in [11. However, for doing computations we need that Mon has more structure; see conditions EGB3 and EGB4 below. This is why we have restricted ourselves to free monoids Mon.

The set $\left\{y_{12} y_{21}, y_{12} y_{23} y_{31}, y_{12} y_{23} y_{34} y_{41}, \ldots\right\}$ in the polynomial ring of Example 1.4 is an infinite $\preceq$-antichain of monomials, hence the $\operatorname{Inc}(\mathbb{N})$-stable ideal generated by it does not have a finite $\operatorname{Inc}(\mathbb{N})$-Gröbner basis. But even in such a setting where not all $G$-stable ideals have finite $G$-Gröbner bases, ideals of interesting $G$ stable varieties may still have such bases. We will derive an algorithm for computing equivariant Gröbner bases under the following two additional assumptions:

EGB3. for all $\pi \in G$ and $m, m^{\prime} \in$ Mon we have $\operatorname{lcm}\left(\pi m, \pi m^{\prime}\right)=\pi \operatorname{lcm}\left(m, m^{\prime}\right)$; and EGB4. for all $f, h \in K[X]$ the set $G f \times G h$ is the union of a finite number of $G$ orbits (where $G$ acts diagonally on $K[X] \times K[X]$ ), and generators of these orbits can be computed effectively.

Note that EGB3 is automatically satisfied if $G$ stabilises the set $X$ of variables. Although this is the only setting that we will need for the application to the twofactor model, future applications may need the greater generality where $X$ is not $G$-stable. Condition EGB4 is of computational importance, as will become clear in Section 2, There we also show in Examples 2.6 and 2.7 that these requirements are not redundant.

Theorem 1.6. Under conditions EGB1, EGB2, EGB3, and EGB4 there exists an algorithm that takes a finite subset $B$ of $K[X]$ as input and that returns a finite $G$-Gröbner basis of the ideal generated by $B$, provided that it terminates. 
There is some overlap in definitions and results by Aschenbrenner, Hillar, and Sullivant [1, 2, 11, by Cohen and his student Emmott [3, 4, and by ourselves; but the three groups pursued distinct goals. In particular, in Section 2 we derive the algorithm from Theorem 1.6 in the non-Noetherian situation where the partial order $\preceq$ is not necessarily a well-quasi-order. This generality is essential in Section 3 , where we apply our algorithm to a conjecture concerning a statistical model to be discussed now.

The Gaussian two-factor model. The Gaussian $k$-factor model with $n$ observed variables consists of all covariance matrices of $n$ jointly Gaussian random variables $X_{1}, \ldots, X_{n}$, the observed variables, consistent with the hypothesis that there exist $k$ further variables $Z_{1}, \ldots, Z_{k}$, the hidden variables or factors, such that the joint distribution of the $X_{i}$ and the $Z_{j}$ is Gaussian and such that the $X_{i}$ are pairwise independent given all $Z_{j}$. This set of covariance matrices turns out to be

$F_{k, n}:=\left\{D+S S^{T} \mid D \in M_{n}(\mathbb{R})\right.$ diagonal and positive definite, and $\left.S \in M_{n, k}(\mathbb{R})\right\}$, where $M_{n, k}(\mathbb{R})$ is the space of real $n \times k$-matrices, and $M_{n}(\mathbb{R})$ is the space of real $n \times n$-matrices. In 9] this model is studied from an algebraic point of view. In particular, the ideal of polynomials vanishing on $F_{k, n}$ is determined for $k=2,3$ and $n \leq 9$. The case where $k=1$ had already been done in [6]. The authors of [9] pose some very intriguing finiteness questions. In particular, one might hope that for fixed $k$ the ideal of $F_{k, n}$ stabilises, as $n$ grows, modulo its natural symmetries coming from simultaneously permuting rows and columns. For $k=1$ this is indeed the case, and for arbitrary $k$ it is true in a weaker, set-theoretic sense 7 . In this paper we prove that the ideals of $F_{2, n}$ stabilise at $n=6$. To state our theorem we denote by $y_{i j}$ the coordinates on the space of symmetric $n \times n$-matrices; we will identify $y_{j i}$ with $y_{i j}$. Recall from [9] that the ideal of $F_{2,5}$ is generated by a single polynomial

$$
P:=\frac{1}{10} \sum_{\pi \in \operatorname{Sym}(5)} \operatorname{sgn}(\pi) y_{\pi(1), \pi(2)} y_{\pi(2), \pi(3)} y_{\pi(3), \pi(4)} y_{\pi(4), \pi(5)} y_{\pi(5), \pi(1)},
$$

called the pentad. The normalisation factor is important only because it ensures that all coefficients are \pm 1 ; indeed, the stabiliser in $\operatorname{Sym}(5)$ of each monomial in the pentad is a dihedral group of order 10 . We consider $P$ an element of $\mathbb{Z}\left[y_{i j} \mid i \geq j\right]$. The ideal of $F_{2,6}$ contains another type of equation: the off-diagonal minor,

$$
M:=\operatorname{det}(y[\{4,5,6\},\{1,2,3\}]) \in \mathbb{Z}\left[y_{i j} \mid i \geq j\right],
$$

the determinant of the square submatrix of $y$ sitting in the lower left corner of $y$. If $f$ is any polynomial in $\mathbb{R}\left[y_{i j} \mid i \geq j\right]$ that vanishes on $F_{2, n}$ and if we regard $f$ as an element of $\mathbb{R}\left[y_{i j} \mid i>j\right]\left[y_{11}, \ldots, y_{n n}\right]$, then each of the coefficients of the monomials in the diagonal variables $y_{i i}$ is a polynomial in the off-diagonal variables that vanishes on $F_{2, n}$, as well. Therefore, the following theorem settles the conjecture of Drton, Sturmfels, and Sullivant, that pentads and off-diagonal minors generate the ideal of $F_{2, n}$ for all $n$; see [9, Conjecture 26].

Theorem 1.7. For any field $K$ and any natural number $n \geq 6$ the kernel $I_{n}(K)$ of the homomorphism $K\left[y_{i j} \mid 1 \leq j<i \leq n\right] \rightarrow K\left[s_{1}, \ldots, s_{n}, t_{1}, \ldots, t_{n}\right]$ determined by $y_{i j} \mapsto s_{i} s_{j}+t_{i} t_{j}$ is generated, as an ideal, by the orbits of $P$ and $M$ under the symmetric group $\operatorname{Sym}(n)$. 
Remark 1.8. In [10] it is proved that $F_{2, n}$ equals the set of all positive definite matrices with the property that every principal $6 \times 6$-minor lies in $F_{2,6}$. Theorem 1.7 implies an analogous statement for the Zariski closures of $F_{2, n}$ and $F_{2,6}$.

We sketch the proof of Theorem 1.7, which appears in Section 3. We put a suitable elimination order on the monomials in $y_{i j}, i, j \in \mathbb{N}, i \geq j$, and report on a computation that yields a finite $\operatorname{Inc}(\mathbb{N})$-Gröbner basis for the determinantal ideal generated by all $3 \times 3$-minors of $y$. Intersecting this $\operatorname{Inc}(\mathbb{N})$-Gröbner basis with the ring in the off-diagonal matrix entries gives Theorem 1.7

\section{AN ALGORITHM FOR EQUiVARIANT GRÖBNER BASES}

We retain the setting of the Introduction: $X$ is a potentially infinite set and Mon is the free commutative monoid generated by $X$, equipped with a term order (EGB1) preserved by the action of a monoid $G$ (EGB2) which also preserves least common multiples (EGB3). Condition EGB4 will be needed only later.

Remark 2.1. Note that $G$ acts by injective maps on Mon by EGB2. It is essential that we allow $G$ to be a monoid rather than a group. Indeed, the image of $G$ in the monoid of injective maps Mon $\rightarrow$ Mon contains no other invertible elements than the identity: If $\pi \in G$ and $m \in$ Mon, then $\pi m \geq m$ since otherwise $m>\pi m>$ $\pi^{2} m>\ldots$ would be an infinite strictly decreasing chain. But then if (the image of) $\pi$ is invertible, we have $\pi(m)>m>\pi^{-1}(m)>\pi^{-2}(m)>\ldots$, another infinite decreasing chain.

We set out to translate familiar notions from the setting of ordinary Gröbner bases to our equivariant setting. In what follows the coeffient in $f$ of $\operatorname{lm}(f)$, the leading coefficient, is denoted $\operatorname{lc}(f)$, and $\operatorname{lt}(f)=\operatorname{lc}(f) \operatorname{lm}(f)$ is the leading term of $f$.

Lemma 2.2. If $I$ is $G$-stable and $B$ is a $G$-Gröbner basis of $I$, then $G B=\{\pi b \mid$ $\pi \in G, b \in B\}$ generates the ideal $I$.

Proof. If not, then take an $f \in I \backslash\langle G B\rangle$ with $\operatorname{lm}(f)$ minimal. Take $b \in B$ and $\pi \in G$ with $\operatorname{lm}(\pi b) \mid \operatorname{lm}(f)$. Subtracting $(\operatorname{lt}(f) / \operatorname{lt}(\pi b)) \pi b$ from $f$ yields an element in $I \backslash\langle G B\rangle$ with leading term strictly smaller than that of $f$, a contradiction.

Algorithm 2.3 (Equivariant remainder). Given $f \in K[X]$ and $B \subseteq K[X]$, proceed as follows: if $\pi \operatorname{lm}(b) \mid \operatorname{lm}(f)$ for some $\pi \in G$ and $b \in B$, then subtract the multiple $(\operatorname{lt}(f) / \operatorname{lt}(\pi b)) \pi b$ of $\pi b$ from $f$, so as to lower the latter's leading monomial. Do this until no such pair $(\pi, b)$ exists. The resulting polynomial is called a $G$-remainder (or an equivariant remainder, if $G$ is fixed) of $f$ modulo $B$.

This procedure is non-deterministic, but necessarily finishes after a finite number of steps, since $\leq$ is a well-order. Any potential outcome is called an equivariant remainder of $f$ modulo $B$.

Definition 2.4 (Equivariant $S$-polynomials). Consider two polynomials $b_{0}, b_{1}$ with leading monomials $m_{0}, m_{1}$, respectively. Let $H$ be a set of pairs $\left(\sigma_{0}, \sigma_{1}\right) \in G \times G$ for which $G b_{0} \times G b_{1}=\bigcup_{\left(\sigma_{0}, \sigma_{1}\right) \in H}\left\{\left(\pi \sigma_{0} b_{0}, \pi \sigma_{1} b_{1}\right) \mid \pi \in G\right\}$. For every element $\left(\sigma_{0}, \sigma_{1}\right) \in H$ we consider the ordinary $S$-polynomial

$$
S\left(\sigma_{0} b_{0}, \sigma_{1} b_{1}\right):=\operatorname{lc}\left(b_{1}\right) \frac{\operatorname{lcm}\left(\sigma_{0} m_{0}, \sigma_{1} m_{1}\right)}{\sigma_{0} m_{0}} \sigma_{0} b_{0}-\operatorname{lc}\left(b_{0}\right) \frac{\operatorname{lcm}\left(\sigma_{0} m_{0}, \sigma_{1} m_{1}\right)}{\sigma_{1} m_{1}} \sigma_{1} b_{1} .
$$


The set $\left\{S\left(\sigma_{0} b_{0}, \sigma_{1} b_{1}\right) \mid\left(\sigma_{0}, \sigma_{1}\right) \in H\right\}$ is called a complete set of equivariant $S$ polynomials for $b_{0}, b_{1}$. It depends on the choice of $H$. Under condition EGB4, $H$ can be chosen finite.

Theorem 2.5 (Equivariant Buchberger criterion). Under the assumptions EGB1, $E G B 2$, and $E G B 3$, let $B$ be a subset of $K[X]$ such that for all $b_{0}, b_{1} \in B$ there exists a complete set of $S$-polynomials each of which has 0 as a $G$-remainder modulo $B$. Then $B$ is a $G$-Gröbner basis of the ideal generated by $G B$.

We will first prove this for ordinary Gröbner bases, and from that deduce the theorem for equivariant Gröbner bases. The proof for the ordinary case is identical to the proof in the case of finitely many variables. We include it for completeness, and also because we have no reference where the result is stated for infinitely many variables.

Proof of Theorem 2.5 in the case $G=\{1\}$. We may and will assume that all elements of $B$ are monic. Let $I$ denote the ideal generated by $B$. If $\operatorname{lm}(B)$ does not generate the ideal $\operatorname{lm}(I)$ in Mon, then there exists a polynomial of the form

$$
f=\sum_{b \in B} f_{b} b
$$

with only finitely many of the $f_{b}$ non-zero, for which $\operatorname{lm}(f)$ is not in the ideal generated by $\operatorname{lm}(B)$. We may choose the expression above such that first, the maximum $m$ of $\operatorname{lm}\left(f_{b} b\right)$ over all $b$ for which $f_{b}$ is non-zero is minimal and second, the number of $b$ with $\operatorname{lm}\left(f_{b} b\right)=m$ is also minimal. The maximum is then attained for at least two values $b_{0}, b_{1}$ of $b$, because otherwise $m$ would be the leading monomial of $f$. Write $m_{i}:=\operatorname{lm}\left(b_{i}\right)$ for $i=0,1$, and let $t_{0}, t_{1}$ be such that $\operatorname{lcm}\left(m_{0}, m_{1}\right)=$ $t_{0} m_{0}=t_{1} m_{1}$. Now $m=\operatorname{lm}\left(f_{b_{0}}\right) m_{0}=\operatorname{lm}\left(f_{b_{1}}\right) m_{1}$ is a multiple of both $m_{0}$ and $m_{1}$, and therefore $\operatorname{lm}\left(f_{b_{0}}\right)$ is divisible by $t_{0}$; set

$$
A:=\frac{\operatorname{lt}\left(f_{b_{0}}\right)}{t_{0}} .
$$

Next consider

$$
S:=S\left(b_{0}, b_{1}\right)=t_{0} b_{0}-t_{1} b_{1},
$$

where we have used that $b_{0}$ and $b_{1}$ are monic. As 0 is a remainder of $S$ modulo $B$ by assumption, we can write $S$ as a sum $\sum_{b \in B} s_{b} b$ with only finitely many non-zero terms that, moreover, satisfy $\operatorname{lm}\left(s_{b} b\right) \leq \operatorname{lm}(S)<\operatorname{lcm}\left(m_{0}, m_{1}\right)$ for all $b$. Then we may rewrite $f$ as

$$
f=f-A\left(S-\sum_{b} s_{b} b\right)=\sum_{b}\left(f_{b}+f_{b}^{\prime}+f_{b}^{\prime \prime}\right) b
$$

where $f_{b}^{\prime}=A s_{b}$ and

$$
f_{b}^{\prime \prime}= \begin{cases}-\operatorname{lt}\left(f_{b_{0}}\right) & \text { if } b=b_{0} \\ \operatorname{lc}\left(f_{b_{0}}\right) \operatorname{lm}\left(f_{b_{1}}\right) & \text { if } b=b_{1} \\ 0 & \text { otherwise }\end{cases}
$$

For all $b \in B$ we have

$$
\begin{aligned}
\operatorname{lm}\left(\left(A s_{b}\right) b\right) & =\operatorname{lm}\left(A s_{b} b\right)<\frac{\operatorname{lm}\left(f_{b_{0}}\right)}{t_{0}} \operatorname{lcm}\left(m_{0}, m_{1}\right) \\
& =\operatorname{lm}\left(f_{b_{0}}\right) m_{0}=m,
\end{aligned}
$$


so for all $b$ we have $\operatorname{lm}\left(f_{b}^{\prime} b\right)<m$. Moreover, $\operatorname{lm}\left(\left(f_{b_{0}}+f_{b_{0}}^{\prime \prime}\right) b_{0}\right)$ is strictly smaller than $m$. Finally, $\operatorname{lm}\left(f_{b_{1}}^{\prime \prime} b_{1}\right)=m$. We conclude that either $\max _{b} \operatorname{lm}\left(\left(f_{b}+f_{b}^{\prime}+f_{b}^{\prime \prime}\right) b\right)$ is strictly smaller than $m$, or else the number of $b$ for which it equals $m$ is smaller than the number of $b$ for which $\operatorname{lm}\left(f_{b} b\right)$ equals $m$. This contradicts the minimality of the expression chosen above.

Proof of Theorem 2.5 using the ordinary Buchberger criterion. We prove that $G B$ is an ordinary Gröbner basis of the ideal that it generates. By the ordinary Buchberger criterion it suffices to verify that for all $b_{0}, b_{1} \in B$ and $\pi_{0}, \pi_{1} \in G$ the $S$ polynomial $S\left(\pi_{0} b_{0}, \pi_{1} b_{1}\right)$ has 0 as a remainder modulo $G B$. By assumption there exists a triple $\left(\sigma_{0}, \sigma_{1}, \pi_{2}\right)$ for which $S\left(\sigma_{0} b_{0}, \sigma_{1} b_{1}\right)$ has 0 as a $G$-remainder modulo $B$-which means that it has 0 as an ordinary remainder modulo $G B$-and for which $\left(\pi_{0} b_{0}, \pi_{1} b_{1}\right)=\left(\pi_{2} \sigma_{0} b_{0}, \pi_{2} \sigma_{1} b_{1}\right)$. Since $G$ preserves least common multiples (EGB3), we have

$$
S\left(\pi_{2} \sigma_{0} b_{0}, \pi_{2} \sigma_{1} b_{1}\right)=\pi_{2} S\left(\sigma_{0} b_{0}, \sigma_{1} b_{1}\right)
$$

and applying $\pi_{2}$ to the entire reduction of $S\left(\sigma_{0} b_{0}, \sigma_{1} b_{1}\right)$ to 0 modulo $G B$ yields a reduction of $S\left(\pi_{0} b_{0}, \pi_{1} b_{1}\right)$ to 0 , as claimed.

The following example shows that EGB3 is not a redundant assumption in Theorem 2.5 .

Example 2.6. Suppose that $X=\left\{x, y, z_{1}, z_{2}, \ldots\right\}$ and that the monoid $G$ is generated by $\operatorname{Inc}(\mathbb{N})$ acting by $\pi z_{i}=z_{\pi i}$ and trivially on $x, y$, together with a single homomorphism $\sigma$ : Mon $\rightarrow$ Mon determined by $\pi x=x, \pi y=x z_{1}$, and $\pi z_{i}=z_{i+1}$ for all $i$. Then $G$ preserves the lexicographic order on Mon for which $z_{i+1}>z_{i}>y>x$ for all $i$. Now consider the set $B=\{y+1\}$. We have

$$
G(y+1) \times G(y+1)=G(y+1, y+1) \cup G\left(y+1, x z_{1}+1\right) \cup G\left(x z_{1}+1, y+1\right),
$$

so we may take $H$ from Definition 2.4 equal to

$$
\{(1,1),(1, \sigma),(\sigma, 1)\} \text {. }
$$

The $S$-polynomial $S(y+1, y+1)$ is zero, and the $S$-polynomials $S\left(y+1, x z_{1}+1\right)$ and $S\left(x z_{1}+1, y+1\right)$ reduce to zero modulo $y+1$ and $\sigma(y+1)=x z_{1}+1$. Hence we have a complete set of $S$-polynomials of $y+1$ with itself that all $G$-reduce to zero modulo $B$. Nevertheless, $B$ is not a $G$-Gröbner basis of the $G$-stable ideal that it generates, since that ideal also contains $S\left(x z_{1}+1, x z_{2}+1\right)=z_{2}-z_{1}$, which does not $G$-reduce to zero modulo $B$.

Here is an example where EGB4 is not fulfilled.

Example 2.7. Let $X=\{x, y\}$, let $G$ be the multiplicative monoid of the positive integers, where $m$ acts by $x \mapsto x^{m}$ and $y \mapsto y^{m}$. Now

$$
G x \times G y=\left\{\left(x^{i}, y^{j}\right) \mid i, j \in \mathbb{Z}_{>0}\right\},
$$

while the diagonal $G$-orbit of the pair $\left(x^{i}, y^{j}\right)$ equals $\left\{\left(x^{a i}, y^{a j}\right) \mid a \in \mathbb{Z}_{>0}\right\}$. Hence $G x \times G y$ is not the union of finitely many $G$-orbits.

However, if we do assume EGB4, then every pair $\left(b_{0}, b_{1}\right)$ has a finite and computable complete set of $S$-polynomials and we have the following theoretical algorithm, alluded to in Theorem 1.6. We do not claim that it terminates, but if it does, then it returns a finite equivariant Gröbner basis by Theorem 2.5 . 
Algorithm 2.8 (Equivariant Buchberger algorithm).

Input: a finite subset $B$ of $K[X]$.

Output (assuming termination): a finite equivariant Gröbner basis of the ideal generated by $G B$.

\section{Procedure:}

(1) $P:=B \times B$;

(2) while $P \neq \emptyset$ do

(a) choose $\left(b_{0}, b_{1}\right) \in P$ and set $P:=P \backslash\left\{\left(b_{0}, b_{1}\right)\right\}$;

(b) compute a finite complete set $\mathcal{S}$ of equivariant $S$-polynomials for $\left(b_{0}, b_{1}\right)$

(c) while $\mathcal{S} \neq \emptyset$ do

(i) choose $f \in \mathcal{S}$ and set $\mathcal{S}:=\mathcal{S} \backslash\{f\}$;

(ii) compute a $G$-remainder $r$ of $f$ modulo $B$;

(3) return $B$.

(iii) if $r \neq 0$ then set $B:=B \cup\{r\}$ and $P:=P \cup(B \times r)$;

Note the order in which $B$ and $P$ are updated: one needs to add $(r, r)$ to $P$, as well. The proof of correctness of this algorithm is straightforward and omitted.

Remark 2.9. If the partial order $\preceq$ on Mon defined in the introduction does not admit infinite antichains, then the equivariant Buchberger algorithm always terminates. Indeed, suppose that the algorithm would not terminate, and let $r_{1}, r_{2}, r_{3}, \ldots$ be the sequence of remainders added consecutively to $B$. Then for all $i<j$ we have $\operatorname{lm}\left(r_{i}\right) \npreceq \operatorname{lm}\left(r_{j}\right)$ since $r_{j}$ is $G$-reduced modulo $r_{i}$. Using the fact that decreasing $\preceq$-chains are finite, one finds an infinite subsequence $r_{i_{1}}, r_{i_{2}}, \ldots$ with $i_{1}<i_{2}<\ldots$ such that $r_{i_{a}} \npreceq r_{i_{b}}$ holds not only for $a<b$ but also for $a>b$. This sequence contradicts the assumption that $\preceq$ does not have infinite antichains.

\section{AN EQUiVARIANT GröBNER BASIS FOR THE TWO-FACTOR MODEL}

Theorem 1.7 will follow from the following result. Let $X=\left\{y_{i j} \mid i, j \in \mathbb{N}, i \geq j\right\}$ be a set of variables representing the entries of a symmetric matrix. We consider the lexicographic monomial order on Mon in which the diagonal variables $y_{i i}$ are larger than all variables $y_{i j}$ with $i>j$, and apart from that $y_{i j} \geq y_{i^{\prime} j^{\prime}}$ if and only if $i>i^{\prime}$ or $i=i^{\prime}$ and $j \geq j^{\prime}$. So, for instance, we have

$$
y_{2,2}>y_{1,1}>y_{5,2}>y_{4,3} \text {. }
$$

Note that this monomial order is compatible with the action of the monoid $\operatorname{Inc}(\mathbb{N})$ of all increasing maps $\mathbb{N} \rightarrow \mathbb{N}$. For any polynomial $p \in K[X]$ let $l(p)$ denote the largest index of $p$, i.e., the largest index appearing in any of the variables in any of the monomials of $p$.

Theorem 3.1. For any field $K$, let $J_{\mathbb{N}}(K)$ be the ideal in $K[X]$ generated by all $3 \times 3$-minors of the matrix $y$ (recall that we identify $y_{j i}$ for $j<i$ with $y_{i j}$ ). Relative to the monomial order $\leq$ the ideal $J_{\mathbb{N}}(K)$ has an $\operatorname{Inc}(\mathbb{N})$-Gröbner basis $B$ consisting of 42 polynomials. The intersection $B \cap K\left[y_{i j} \mid i>j\right]$ is an $\operatorname{Inc}(\mathbb{N})$-Gröbner basis of $J_{\mathbb{N}}(K) \cap K\left[y_{i j} \mid i>j\right]$ consisting of 20 polynomials. The largest indices and the degrees of the elements in these bases are summarised in Table 3.1 .

Remark 3.2. The polynomial with largest index 5 in the $\operatorname{Inc}(\mathbb{N})$-Gröbner basis $B \cap K\left[y_{i j} \mid i>j\right]$ is the pentad $P$. The five degree-3 polynomials with largest index 6 
TABLE 1. Degrees of polynomials in the $\operatorname{Inc}(\mathbb{N})$-Gröbner basis $B$ of $J_{\mathbb{N}}(K)$, grouped according to largest index. The first row records the largest index, the second row the number of polynomials in $B$ with that largest index, the third row their degrees with multiplicities written as exponents, the fourth row counts the number of polynomials containing only variables $y_{i j}$ with $i>j$, and the fifth row records their degrees.

\begin{tabular}{|l|lllllll|}
\hline$l(p)$ & 3 & 4 & 5 & 6 & 7 & 8 & 9 \\
\hline$\# p \in B$ & 1 & 6 & 11 & 10 & 8 & 5 & 1 \\
degrees & $3^{1}$ & $3^{6}$ & $3^{10} 5^{1}$ & $3^{5} 5^{5}$ & $5^{8}$ & $5^{5}$ & $5^{1}$ \\
$\# p \in B \cap K\left[y_{i j} \mid i>j\right]$ & & & 1 & 5 & 8 & 5 & 1 \\
degrees & & & $5^{1}$ & $3^{5}$ & $5^{8}$ & $5^{5}$ & $5^{1}$ \\
\hline
\end{tabular}

in that Gröbner basis form the $\operatorname{Sym}(\mathbb{N})$-orbit of the off-diagonal minor $M$. All 19 remaining polynomials are already in the $\operatorname{Inc}(\mathbb{N})$-stable ideal generated by these polynomials; this latter statement also follows from the result in [9] that at least up to $n=9$ the ideal of the two-factor model is generated by pentads and off-diagonal minors. The complete basis $B$ is available from http://www.win.tue.nl/ jdraisma/ index.php?location=publications.

Remark 3.3. A Gröbner basis of the ideal of the two-factor model $F_{2, n}$ relative to circular term orders was already found in [14. The proof involves general techniques for determining the ideal of secant varieties, especially of toric varieties; see also [13. The Gröbner basis found there, however, does not stabilise as $n$ grows - and indeed, circular term orders are not compatible with the action of $\operatorname{Inc}(\mathbb{N})$. It would be interesting to find a direct translation between Sullivant's Gröbner basis and ours.

Theorem 3.1 implies Theorem 1.7.

Proof of Theorem 1.7. It is well known that the $(k+1) \times(k+1)$-minors of the symmetric matrix $\left(y_{i j}\right)_{i, j=1, \ldots, n}$ generate the ideal of all polynomials vanishing on all rank- $k$ matrices (for a recent combinatorial proof of this fact, see 13, Example 4.12]; in characteristic 0 this fact is known as the Second Fundamental Theorem for the orthogonal group). Hence the ideal $I_{n}(K)$ of Theorem 1.7 is the intersection of the ideal $J_{n}$ generated by the $3 \times 3$-minors of $\left(y_{i j}\right)_{i, j=1, \ldots, n}$ with the ring $K\left[y_{i j} \mid\right.$ $i>j$ ]. Theorem 3.1 implies that one obtains a Gröbner basis of $J_{n}$, relative to the restriction of the monomial order on $K\left[y_{i j} \mid i, j \in \mathbb{N}, i \geq j\right]$ to $K\left[y_{i j} \mid 1 \leq j<i \leq n\right]$ by applying all increasing maps $\{1, \ldots, l(p)\} \rightarrow\{1, \ldots, n\}$ to all $p \in B \cap K\left[y_{i j} \mid\right.$ $i>j]$ with $l(p) \leq n$. Such an increasing map can be extended to an element of $\operatorname{Sym}(n)$, and Remark 3.2 concludes the proof.

We conclude with some remarks on the computation that proved Theorem 3.1. First we need to verify EGB4.

Lemma 3.4. For all $b_{0}, b_{1} \in K\left[y_{i j} \mid i, j \in \mathbb{N}, i \geq j\right]$ the set $\left(\operatorname{Inc}(\mathbb{N}) b_{0}\right) \times\left(\operatorname{Inc}(\mathbb{N}) b_{1}\right)$ is the union of a finite number of $\operatorname{Inc}(\mathbb{N})$-orbits.

Proof. Consider all pairs $\left(S_{0}, S_{1}\right)$ of sets $S_{0}, S_{1} \subseteq \mathbb{N}$ with $\left|S_{i}\right|=l\left(b_{i}\right)$ for which $S_{0} \cup S_{1}$ is an interval of the form $\{1, \ldots, k\}$ for some $k$, which is then at most $l\left(b_{0}\right)+l\left(b_{1}\right)$. Note that there are only finitely many such pairs $\left(S_{0}, S_{1}\right)$. For each 
such pair let $\left(\pi_{0}, \pi_{1}\right)$ be a pair of elements of $\operatorname{Inc}(\mathbb{N})$ such that $\pi_{i}$ maps $\left\{1, \ldots, l\left(b_{i}\right)\right\}$ onto $S_{i}$; it is irrelevant how $\pi$ acts on the rest of $\mathbb{N}$. Then we have

$$
\operatorname{Inc}(\mathbb{N}) b_{0} \times \operatorname{Inc}(\mathbb{N}) b_{1}=\bigcup_{\left(S_{0}, S_{1}\right)} \operatorname{Inc}(\mathbb{N})\left(\pi_{0} b_{0}, \pi_{1} b_{1}\right),
$$

where the union is over all pairs $\left(S_{0}, S_{1}\right)$ as above.

Computational proof of Theorem 3.1. The 42 polynomials of $B$ were constructed by computing a Gröbner basis for $J_{9}(\mathbb{Q})$ with Singular and retaining only those polynomials $p$ for which the set of indices occurring in their variables form an interval of the form $\{1, \ldots, k\}$ with $k \leq 9$. All elements of $B$ are monic and have integral coefficients (in fact, equal to \pm 1 except for the $3 \times 3$-minor with largest index 3, which has a coefficient 2). By the equivariant Buchberger criterion and the proof of Lemma 3.4, we need only $\operatorname{Inc}(\mathbb{N})$-reduce modulo $B$ all $S$-polynomials of pairs $\left(\pi_{0} b_{0}, \pi_{1} b_{1}\right)$ with $b_{0}, b_{1} \in B$ and $\pi_{i}:\left\{1, \ldots, l\left(b_{i}\right)\right\} \rightarrow \mathbb{N}$ increasing and such that im $\pi_{0} \cup \operatorname{im} \pi_{1}=\{1, \ldots, k\}$ for some $k$. For instance, for $b_{0}=b_{1}=b$ equal to the polynomial in $B$ with largest index 9 , we have to $\operatorname{Inc}(\mathbb{N})$-reduce $S\left(\pi_{0} b, \pi_{1} b\right)$ modulo $B$ for all increasing maps $\pi_{0}, \pi_{1}:\{1, \ldots, 9\} \rightarrow\{1, \ldots, 18\}$ whose image union is an interval $\{1, \ldots, k\}$. However, if $k=17$ or $k=18$, then $\pi_{0} b$ and $\pi_{1} b$ turn out to have leading monomials with gcd 1 , so these cases can be skipped. This reduces the theorem to a finite computation involving polynomials with largest indices up to 16 , which we have implemented directly in C. Finally, to deduce the result for all base fields - and to speed up the computation - we used the following argument. Since $\operatorname{Inc}(\mathbb{N}) B \cap K\left[y_{i j} \mid 1 \leq j \leq i \leq n\right]$ is a subset of the ideal of $3 \times 3$-minors, it is a Gröbner basis if and only if the ideal generated by $\operatorname{lm}(B)$ has the same Hilbert series as the ideal generated by $3 \times 3$-minors. Since this Hilbert series is known and does not depend on the field [5], we may do all our computations over one field and conclude that it holds over all fields. We have verified the equivariant Buchberger criterion over $\mathbb{F}_{2}$, which made the computation slightly faster than working over $\mathbb{Q}$.

\section{ACKNOWLEDGMENTS}

We thank Jan Willem Knopper and Rudi Pendavingh for motivating discussions on alternative computations that would prove Theorem 3.1. We also thank the referees for suggestions on improving the exposition. Finally, we thank Alexei Krasilnikov for pointing out the references [3] and [4].

\section{REFERENCES}

[1] Matthias Aschenbrenner and Christopher J. Hillar. Finite generation of symmetric ideals. Trans. Amer. Math. Soc., 359(11):5171-5192, 2007. MR2327026 (2008g:13030)

[2] Matthias Aschenbrenner and Christopher J. Hillar. An algorithm for finding symmetric Gröbner bases in infinite dimensional rings. ISSAC 2008, 117-123, ACM, New York, 2008. MR2500387

[3] Daniel E. Cohen. On the laws of a metabelian variety. J. Algebra, 5:267-273, 1967. MR0206104 (34:5929)

[4] Daniel E. Cohen. Closure relations, Buchberger's algorithm, and polynomials in infinitely many variables. In Computation theory and logic, volume 270 of Lect. Notes Comput. Sci., pages 78-87, 1987. MR 907514 (89a:68113)

[5] Aldo Conca. Gröbner bases of ideals of minors of a symmetric matrix. J. Algebra, 166(2):406421, 1994. MR1279266 (95g:13012) 
[6] Jesús A. de Loera, Bernd Sturmfels, and Rekha R. Thomas. Gröbner bases and triangulations of the second hypersimplex. Combinatorica, 15:409-424, 1995. MR1357285 (97b:13035)

[7] Jan Draisma. Finiteness for the $k$-factor model and chirality varieties. Adv. Math., 223:243256, 2010. MR2563217

[8] Vesselin Drensky and Roberto La Scala. Gröbner bases of ideals invariant under endomorphisms. J. Symb. Comput., 41(7):835-846, 2006. MR.2232204(2007m:16023)

[9] Mathias Drton, Bernd Sturmfels, and Seth Sullivant. Algebraic factor analysis: tetrads, pentads and beyond. Probab. Theory Relat. Fields, 138(3-4):463-493, 2007. MR2299716 (2008f:62086)

[10] Mathias Drton and Han Xiao. Finiteness of small factor analysis models. personal communication, 2008.

[11] Chris J. Hillar and Seth Sullivant. Finite Gröbner bases in infinite dimensional polynomial rings and applications. Preprint, available from http://arxiv.org/abs/0908.1777, 2009.

[12] Bernd Sturmfels. Gröbner bases and Stanley decompositions of determinantal ideals. Math. Z., 205(1):137-144, 1990. MR.1069489 (91m:14076)

[13] Bernd Sturmfels and Seth Sullivant. Combinatorial secant varieties. Pure Appl. Math. Q., 2(3):867-891, 2006. MR2252121 (2007h:14082)

[14] Seth Sullivant. A Gröbner basis for the secant ideal of the second hypersimplex. J. Commut. Algebra 1(2):327-338. MR2504939 (2010d:13033)

Department of Mathematics and Computer Science, Technische Universiteit Eindhoven, P.O. Box 513, 5600 MB Eindhoven, The Netherlands

E-mail address: aeb@cwi.nl

Department of Mathematics and Computer Science, Technische Universiteit Eindhoven, P.O. Box 513, 5600 MB Eindhoven, The Netherlands and Centrum voor Wiskunde en Informatica, Amsterdam, The Netherlands

E-mail address: j.draisma@tue.nl 